\title{
Blood lead levels of contemporary Japanese children
}

\author{
Jun Yoshinaga $\cdot$ Mai Takagi $\cdot$ Kumiko Yamasaki $\cdot$ \\ Sayaka Tamiya $\cdot$ Chiho Watanabe $\cdot$ \\ Masayuki Kaji
}

Received: 20 January 2011/Accepted: 4 April 2011/Published online: 23 April 2011

(c) The Japanese Society for Hygiene 2011

\begin{abstract}
Objectives Lead concentrations in whole blood $(\mathrm{BPb})$ of Japanese children were measured to obtain insight into the sources of variation and to estimate health risk.

Methods Blood samples were taken from 352 children (aged 1-14 years) at pediatric clinics/hospitals in 3 regions of Japan (Tokyo, Shizuoka, and Osaka) during 2005-2006 (Shizuoka only) and 2008-2010 (3 regions), and BPb was measured by inductively coupled plasma mass spectrometry after acid digestion.

Results Geometric mean $\mathrm{BPb}$ of the 352 children was $1.07 \mu \mathrm{g} / \mathrm{dL}$, among the lowest in the world. Based on this result, the probability of exceeding $\mathrm{BPb} 10 \mu \mathrm{g} / \mathrm{dL}$, the action level proposed by the US Centers for Disease Control and Prevention, in general Japanese children was estimated to be negligible. There was a significant negative correlation between $\mathrm{BPb}$ and age, while sex difference was not significant. $\mathrm{BPb}$ was significantly lower in subjects in Tokyo than in the other two regions. Age-adjusted mean $\mathrm{BPb}$ was significantly higher in children with a family member who smoked in their presence than in those who did not have such a family member, indicating that passive smoking had a significant effect on $\mathrm{BPb}$.
\end{abstract}

J. Yoshinaga $(\bowtie) \cdot$ M. Takagi · K. Yamasaki · S. Tamiya Department of Environmental Studies, University of Tokyo, Kashiwanoha 5-1-5, Kashiwa, Chiba 270-8563, Japan e-mail: junyosh@k.u-tokyo.ac.jp

C. Watanabe

Department of Human Ecology,

School of International Health, University of Tokyo,

Hongo 7-3-1, Bunkyo, Tokyo 113-0033, Japan

M. Kaji

Shizuoka Public Health Center, Joto-cho 24-1,

Aoi, Shizuoka, Shizuoka 420-0846, Japan
Conclusions Health risk of lead exposure is minimal in Japanese children. To lower the exposure level further, reduction of passive smoking is suggested as one of the effective measures.

Keywords Blood · Lead C Children · Variation · Passive smoking

\section{Introduction}

Lead $(\mathrm{Pb})$ is a well-known neurotoxicant. A number of epidemiologic studies done since the 1980s have revealed that $\mathrm{Pb}$ impairs normal development of cognitive functions of children [1]. Exposure to $\mathrm{Pb}$ has been related to lower intelligence quotient (IQ) [2], poor academic achievement [3], and delinquent behavior [4]. Moreover, concern has recently been raised that adverse effects are seen at exposure levels lower than were considered safe in the past [5, 6]. Schwartz [7] and Lanphear et al. [8] suggested that there is no threshold for $\mathrm{Pb}$ exposure below which no adverse effect on cognitive development was observed.

Lead concentration in whole blood (BPb) has long been considered a reliable indicator of relatively short-term (approximately 1 month [1]) exposure to $\mathrm{Pb}$, and it was measured to represent exposure level in most of the previous epidemiologic studies on $\mathrm{Pb}$ and cognitive functions of children. $\mathrm{BPb}$ has also been measured routinely for biomonitoring of $\mathrm{Pb}$ exposure of children in many countries.

However, in Japan, BPb in children has not been extensively measured. To the best of our knowledge, there are only two reports on children's blood, taken in Shizuoka in 1993 and in 2004-2005 [9-11]. This scarcity of data may be related to the notion that Japan has been one of the 
least $\mathrm{Pb}$-contaminated countries in the world, because early phase-out of leaded gasoline was achieved by the mid1980 s and it is not the custom to paint residences; residential paint used in some countries in the past was $\mathrm{Pb}$ based and a well-known source of $\mathrm{Pb}$ poisoning [12] and elevated $\mathrm{BPb}$ [13] of children in such countries. In agreement with this assumption, $\mathrm{BPb}$ data obtained in Shizuoka (mean 3.16 and $1.55 \mu \mathrm{g} / \mathrm{dL}$ in 1993 and 2004-2005, respectively) demonstrated lower $\mathrm{BPb}$ than in other countries.

However, since no threshold for cognitive effects has been postulated, $\mathrm{BPb}$ should be monitored in Japan more extensively, even though $\mathrm{BPb}$ levels of Japanese children are expected to be low. In this paper we report $\mathrm{BPb}$ of Japanese children. Variation due to physiological (age and sex) and regional factors and passive smoking status were examined. Information on the source of variation may be helpful in identifying the source of exposure to $\mathrm{Pb}$ in Japanese children.

\section{Materials and methods}

\section{Collaborating pediatricians}

Eight pediatricians from 3 regions of Japan (Tokyo, Shizuoka, and Osaka) in addition to one of the authors of this study (M.K.) collaborated in blood sampling. There were six collaborating pediatricians in Tokyo from one general hospital and five pediatric clinics, two in Shizuoka (including M.K.) from one general hospital, and one in Osaka from a general hospital.

\section{Contamination check}

Since BPb of Japanese children was expected to be low, any $\mathrm{Pb}$ contamination derived from blood sampling and storage can be critical. Contamination levels of stainlesssteel needles, syringes, and blood collection tubes were examined prior to blood sampling.

The water used in this study was purified using a Millipore $^{\mathrm{R}}$ Elix/Academic A10 system. Purified water taken in polypropylene (PP) tube, which had been cleaned of $\mathrm{Pb}$ contamination by soaking in $6 \mathrm{~mol} / \mathrm{L}$ nitric acid for 1 week followed by vigorous rinsing with purified water, was drawn with stainless-steel needle and syringe, and the aspirated water was taken in another cleaned PP tube. The needles and syringes were from two manufacturers and were used in the collaborating clinics/hospitals. Concentration of $\mathrm{Pb}$ in the water was measured by inductively coupled plasma mass spectrometry (ICPMS, Agilent 7500ce; Agilent Technologies Japan Ltd., Tokyo) as described below.
Three types of blood collection tube were tested for $\mathrm{Pb}$ contamination. The tubes were from two manufacturers: two of the three tubes had anticoagulants [ethylenediamine tetraacetic acid (EDTA) $2 \mathrm{~K}$ and heparin $\mathrm{Na}$ ], and one did not have anticoagulant. Into eight to nine tubes of each type was added $2 \mathrm{~mL}$ purified water, followed by gentle agitation and standing for $30 \mathrm{~min}$ at room temperature. Then, $\mathrm{Pb}$ concentration in the water was measured by ICPMS. The tube with acceptable $\mathrm{Pb}$ contamination was selected and distributed to the collaborating pediatricians.

\section{Blood sampling}

Blood sampling by collaborating pediatricians was carried out in two separate periods: one in 2005-2006 in Shizuoka only, and the other in 2008-2010 in Shizuoka, Tokyo, and Osaka regions. The purpose and procedure of this study were explained to the child's caretaker by the collaborating pediatrician. Children aged 1 year and older, and whose caretaker voluntarily agreed to participation and gave written consent, were the subjects of this study. Blood was sampled from the subject child by the collaborating pediatrician at the time of blood sampling for diagnosis and other routine examination. The diagnoses of most of the sick subject children included acute infectious diseases, such as upper respiratory diseases. Apparently healthy children who underwent blood sampling for allergy testing were another major group of subjects. In total, 352 children donated blood sample for this study: 161 from Tokyo, 34 from Osaka, and 187 from Shizuoka.

After sampling, blood in the collection tube was immediately frozen and sent to University of Tokyo for $\mathrm{Pb}$ analysis. Information on passive smoking of children was obtained from the caretaker by a brief questionnaire at the time of blood sampling. The caretaker was asked if there was smoking person(s) in their home. If yes, the caretaker was further asked whether the smoking family member(s) smoked in the presence of the child. This information, as well as the age and sex of the subject children, were analyzed in relation to $\mathrm{BPb}$ concentration.

\section{Blood $\mathrm{Pb}$ analysis}

Blood samples were digested by the "double vessel method" [14]. To a $0.5 \mathrm{~mL}$ whole blood sample taken in a $4.5 \mathrm{~mL}$ volume in a capped polytetrafluoroethylene (PTFE) vessel (N-3; San-ai Kagaku, Nagoya), $0.5 \mathrm{~mL}$ ultrapure nitric acid (AA-100; Tama Chemicals Co. Ltd., Kawasaki) was added with digestion in a sealed, stainless-steel jacket at $140^{\circ} \mathrm{C}$ for $4 \mathrm{~h}$. After cooling, the digested sample was diluted to $30 \mathrm{~g}$ in a PP tube with purified water with thallium (Tl, atomic absorption grade; Kanto Chemical Co. Inc., Tokyo) as an internal standard to final concentration 
of $1 \mathrm{ng} / \mathrm{g}$. All operations were done under a compact clean booth supplied with high-efficiency particulate air (HEPA)filtered air. All of the PP tubes and PTFE vessels were soaked in $6 \mathrm{~mol} / \mathrm{L}$ nitric acid for at least 1 week before use.

Lead concentration was measured by internal standardization. Working standard solution, prepared from commercial multi-element standard solution (SPEX XSTC622, USA), contained $1 \mathrm{ng} / \mathrm{g} \mathrm{Pb}$ and $0.3 \mathrm{~mol} / \mathrm{L}$ nitric acid. The operational conditions for ICPMS were as follows: radiofrequency (RF) power $1.5 \mathrm{~kW}$, plasma gas flow rate $12 \mathrm{~L} / \mathrm{min}$, carrier gas flow rate $0.84 \mathrm{~L} / \mathrm{min}$, make-up gas flow rate $0.45 \mathrm{~L} / \mathrm{min}$, monitored $\mathrm{m} / \mathrm{z}, 206,207$, and 208 for $\mathrm{Pb}$ and 205 for $\mathrm{Tl}$, and integration time $0.3 \mathrm{~s}$ for each $\mathrm{m} / \mathrm{z}$.

Analytical quality control

Each batch of blood digestion was composed of one procedural blank, one whole blood reference material (RM, Seronorm $^{\mathrm{TM}}$ Trace Elements Whole Blood L-1; Sero, Norway), and 5-15 samples from subject children. The analytical result of RM was checked, and when significant deviation from the reference range was found, remeasurement was undertaken.

\section{Statistical analysis}

Lead concentration in whole blood had a skewed distribution. Since the distribution of log-transformed values was regarded as normal (Kolmogorov-Smirnov test), logtransformed values were used in statistical analyses. $t$-Test, one-way analysis of variance (ANOVA) with Scheffé's multiple-comparison procedure, Pearson correlation analysis, and analysis of covariance (ANCOVA) were carried out by using the SPSS statistical package (version 12.0J) for PC.

The probability of exceeding a certain, prefixed $\mathrm{BPb}$ level [e.g., the US Centers for Disease Control and Prevention (CDC) action level] was estimated by generating 100,000 random hypothetical $\mathrm{BPb}$ values according to the observed $\mathrm{BPb}$ distribution in this study and counting the number of trials that exceeded the prefixed $\mathrm{BPb}$ level using Crystal Ball (Decisioneering Inc., USA), which was similar to the method employed by Kobayashi and Yoshida [11].

This study was approved by the Ethical Committee of the University of Tokyo and collaborating clinics/hospitals.

\section{Results}

The $\mathrm{Pb}$ contamination level of the three blood collection tubes tested was $0.01,0.05$, and $0.6 \mathrm{ng} /$ tube. We selected the tube with $0.05 \mathrm{ng} /$ tube contamination for this study because the tube with the lowest value $(0.01 \mathrm{ng} / \mathrm{tube}) \mathrm{did}$ not contain anticoagulant: blood clotting was considered problematic for taking aliquots of homogeneous, representative subsamples from each tube for digestion. Possible $\mathrm{Pb}$ contamination from stainless-steel needles and/or syringes for blood sampling was detected to be $0.006 \mathrm{ng}$. Therefore, the total $\mathrm{Pb}$ contamination during blood sampling and storage was expected to be $0.056 \mathrm{ng}$.

During the course of $\mathrm{BPb}$ analysis of 352 samples, 24 subsamples of RM were analyzed, and the mean and standard deviation of $\mathrm{Pb}$ concentration was $2.61 \pm$ $0.09 \mu \mathrm{g} / \mathrm{dL}$. This RM (Seronorm ${ }^{\mathrm{TM}}$ Trace Elements Whole Blood L-1) has two reference values: $2.76 \mu \mathrm{g} / \mathrm{dL}$ (range $2.62-2.90 \mu \mathrm{g} / \mathrm{dL}$ ) determined by inductively coupled plasma (ICP)-sector field MS and $2.1 \mu \mathrm{g} / \mathrm{dL}(1.7-2.5 \mu \mathrm{g} / \mathrm{dL})$ by graphite furnace atomic absorption spectrometry (GFAAS), and our average value $(2.61 \mu \mathrm{g} / \mathrm{dL})$ was intermediate between these two ranges. In addition, the coefficient of variation of our analyses was as small as 3.4\%, demonstrating good reproducibility of analysis. These results for RM demonstrated the accuracy of our blood $\mathrm{Pb}$ analysis. Procedural blanks rarely exceeded the limit of detection of our ICPMS measurement $(0.0001 \mathrm{ng} / \mathrm{g}$ or $0.003 \mathrm{ng}$ ).

Figure 1 shows a histogram of the $\mathrm{BPb}$ values for 352 subject children (184 boys, 167 girls, 1 unknown) determined in this study. As seen from this histogram, the distribution was skewed toward higher values, indicating log-normal distribution. Geometric mean (GM) $\mathrm{BPb}$ concentrations are reported in Table 1.

Based on the observed distribution of $\mathrm{BPb}$ shown in Fig. 1, Monte Carlo simulation revealed that the number of trials that exceeded $4 \mu \mathrm{g} / \mathrm{dL}$ (the tentative threshold value estimated from Surkan et al. [5] and Jusko et al. [6]) and $10 \mu \mathrm{g} / \mathrm{dL}$ (the US CDC action level) was 75 and 0 , respectively, out of 100,000 random trials.

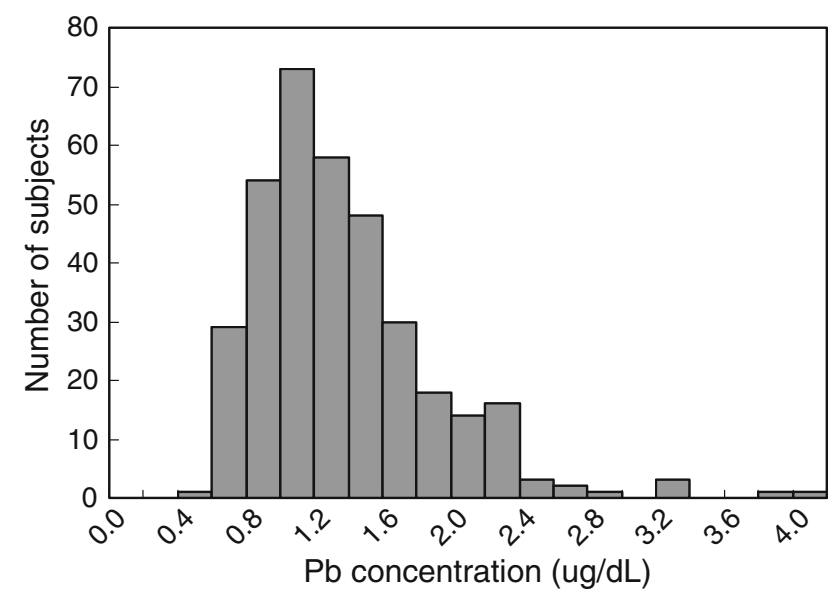

Fig. 1 Histogram of $\mathrm{Pb}$ concentration values in blood samples of 352 children 
When sex- and age-related variation was examined for the whole dataset $(n=352)$, there was a significant negative correlation between $\mathrm{BPb}$ and age $(r=-0.193$, $p<0.001)$. Therefore, in the following comparisons, age was considered as a potential covariate in the models.

Geometric mean [geometric standard deviation (GSD) in parenthesis] $\mathrm{BPb}$ was $1.11 \mu \mathrm{g} / \mathrm{dL}(1.47)$ and $1.04 \mu \mathrm{g} / \mathrm{dL}$ (1.55) for males and females, respectively, and $t$ test did not find significant sex difference (no sex difference was observed for mean age).

In this study, three regions of Japan were selected, i.e., Tokyo, Shizuoka, and Osaka. For Shizuoka region, blood sampling was done in two different periods: one in 2005-2006 $(n=132)$ and the other in 2008-2010 $(n=55)$. Sampling in Tokyo and Osaka was done in 2008-2010 only. For Shizuoka samples, there was no significant difference in mean BPb (GM 1.18 and $1.07 \mu \mathrm{g} / \mathrm{dL}$ in 2005-2006 and 2008-2010, respectively) or age (7.1 and 7.7 years) between 2005-2006 and 2008-2010; therefore, these two periods are combined in the following analyses.

Table 1 Average lead concentrations in blood samples from 3 regions

\begin{tabular}{lrll}
\hline & $N$ & $\begin{array}{l}\text { Age } \\
(\text { years })^{* *}\end{array}$ & $\begin{array}{l}\text { Blood } \mathrm{Pb} \text { concentration } \\
(\mathrm{GM}, \mu \mathrm{g} / \mathrm{dL})^{* *}\end{array}$ \\
\hline Tokyo & 131 & $6.2 \pm 3.8^{\mathrm{a}}$ & $0.940(1.42)^{\#}$ \\
Osaka & 34 & $4.8 \pm 3.6$ & $1.24(1.41)$ \\
Shizuoka & 187 & $7.3 \pm 3.6^{\mathrm{a}, \#}$ & $1.15(1.56)$ \\
Total & 352 & $6.6 \pm 3.8$ & $1.07(1.51)$ \\
\hline
\end{tabular}

a Age of two subjects was missing for Tokyo and Shizuoka

** Variation in age and blood $\mathrm{Pb}$ concentrations due to the regions was significant (ANOVA, $p<0.01$ and $<0.001$, respectively)

\# Significantly different from the other two regions (Scheffé's multiple-comparison procedure)
Geometric means and GSDs of $\mathrm{BPb}$ are also presented in Table 1, divided into the 3 regions. Mean $\mathrm{BPb}$ significantly varied from region to region: the lowest GM was seen in Tokyo and the highest in Osaka. However, mean age of subjects was also significantly different by region. ANCOVA using age as covariate still found significantly lower BPb in Tokyo than in Shizuoka and Osaka. Table 2 presents a comparison of $\mathrm{BPb}$ by age group and region. ANOVA within each age group found significant variation due to region, and regional difference in GM in each age group was only significant for the pair Tokyo-Shizuoka $(p<0.05$, Table 2$)$. Since there were 6 collaborating pediatricians in Tokyo, geographic variation within Tokyo was also examined; however, no significant variation was found after controlling for age (data not shown).

The subject children were divided into three groups according to passive smoking status: no smoking family member ( $n=162$ ), smoker present but does not smoke in the presence of child ( $n=115)$, and smoker present and smokes in the presence of child $(n=64)$. Data for passive smoking were missing for 11 subjects.

ANOVA found marginally nonsignificant variation in $\mathrm{BPb}$ concentration due to passive smoking status $(F=2.349, p=0.097)$. However, mean age was significantly different by passive smoking status (higher mean age in children with family members who smoked in the presence of child). Therefore, ANCOVA analysis was applied controlling for age. Figure 2 shows age-adjusted $\mathrm{BPb}$ according to passive smoking status. Children with passive smoking had significantly higher $\mathrm{BPb}$ (adjusted $\mathrm{BPb} 1.21 \mu \mathrm{g} / \mathrm{dL})$ than children who were expected to be passive smoking-free (1.07 and $1.02 \mu \mathrm{g} / \mathrm{dL})$. When the same analysis was applied separately to each region, the results were similar, although not necessarily identical, to that of the total dataset. For Shizuoka, the result was the same, i.e., higher $\mathrm{BPb}$ in children with passive smoking.

Table 2 Comparison of blood $\mathrm{Pb}$ concentration $(\mu \mathrm{g} / \mathrm{dL})$ of children by age group and region

\begin{tabular}{|c|c|c|c|c|c|c|c|c|c|}
\hline \multirow[t]{2}{*}{ Age range (years) } & \multicolumn{2}{|c|}{ Tokyo } & \multicolumn{2}{|c|}{ Osaka } & \multicolumn{2}{|c|}{ Shizuoka } & \multicolumn{2}{|c|}{ Total } & \multirow[t]{2}{*}{ ANOVA } \\
\hline & $n$ & GM (GSD)* & $n$ & GM (GSD) & $n$ & GM (GSD) & $n$ & GM (GSD) & \\
\hline $1-3$ & 40 & $1.09(1.42)^{\mathrm{a} \alpha}$ & 17 & $1.34(1.42)^{\mathrm{a}}$ & 34 & $1.34(1.59)^{\mathrm{b}}$ & 91 & $1.22(1.50)^{\alpha}$ & $p<0.05$ \\
\hline $4-6$ & 36 & $0.923(1.40)^{\mathrm{a} \alpha}$ & 8 & $1.30(1.34)^{\mathrm{a}}$ & 47 & $1.15(1.60)^{\mathrm{b}}$ & 91 & $1.06(1.53)^{\alpha}$ & $p<0.05$ \\
\hline $7-10$ & 22 & $0.847(1.30)^{\mathrm{a} \beta}$ & 6 & $1.11(1.41)^{\mathrm{a}}$ & 53 & $1.12(1.54)^{\mathrm{b}}$ & 81 & $1.04(1.50)^{\alpha}$ & $p<0.05$ \\
\hline$>11$ & 31 & $0.833(1.42)^{\mathrm{a} \beta}$ & 3 & $0.889(1.45)^{\mathrm{a}}$ & 51 & $1.07(1.50)^{\mathrm{b}}$ & 85 & $0.970(1.49)^{\beta}$ & $p<0.05$ \\
\hline ANOVA $^{\#}$ & & $p<0.001$ & & NS & & NS & & $p<0.01$ & \\
\hline
\end{tabular}

Significant difference is present between GMs with different alphabet (regional difference) and Greek letters (age grade) (Scheffé's multiplecomparison procedure)

The total number of subjects was 348 in this table because there were 4 children without age information in the 352 children

NS not significant

* Geometric mean and geometric standard deviation in parenthesis

\# Analysis of variance 


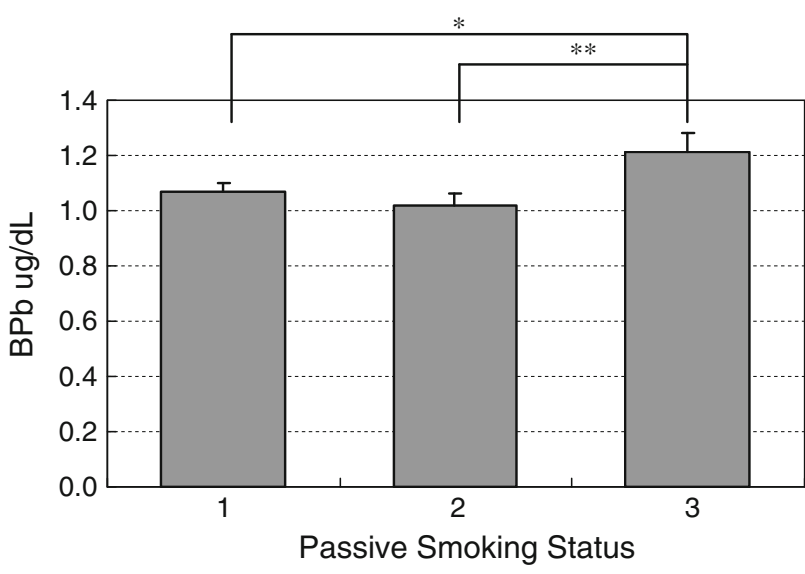

Fig. 2 Comparison of age-adjusted geometric mean $\mathrm{BPb}$ by passive smoking status: status 1 no smoking family member $(n=162)$, status 2 smoking family member present but does not smoke in the presence of child ( $n=115)$, status 3 smoking family member present and smokes in the presence of child $(n=64)$. Standard error of adjusted mean is indicated on the bar graph. * and ** indicate significant difference at $p<0.05$ and $p<0.01$, respectively

However, for Tokyo, the tendency was similar but did not reach statistical significance. For the Osaka subsample, the correlation between $\mathrm{BPb}$ and age was weak and ANCOVA could not be applied. ANOVA did not find significant variation in $\mathrm{BPb}$ according to passive smoking status in Osaka. Lack of significance in Tokyo and Osaka was probably because of loss of statistical power due to reduction of sample size.

\section{Discussion}

Because $\mathrm{BPb}$ analysis involves trace analysis of $\mathrm{Pb}$, which is prone to external contamination, we paid utmost attention to contamination control and analytical quality assurance. The total amount of contamination estimated from blood sampling/storage devices was $0.056 \mathrm{ng}$, which was $<0.3 \%$ of the expected amount of $\mathrm{Pb}$ in $2 \mathrm{~mL}$ blood samples assuming $\mathrm{BPb}$ of $1 \mu \mathrm{g} / \mathrm{dL}$. Procedural blanks during digestion of blood sample were typically undetectable $(<0.003 \mathrm{ng})$ and negligible. Thus, our present $\mathrm{BPb}$ analysis was done under well-controlled conditions in terms of external contamination. In addition, the result of RM analyses was satisfactory in terms of precision and trueness of the analysis. All of our quantitative evaluation of contamination during blood sampling and accuracy of $\mathrm{BPb}$ analysis demonstrated the reliability of our $\mathrm{BPb}$ analysis.

As shown in Table 1, average $\mathrm{BPb}$ of the present subjects was $1.07 \mu \mathrm{g} / \mathrm{dL}(\mathrm{GM})$, among the lowest in the world. Table 3 summarizes some of the recent data on $\mathrm{BPb}$ of children reported in other countries, though year of sampling and age of subject children vary among these reports [15-21]. The lower BPb in Japanese children may be due to lack of apparent $\mathrm{Pb}$ exposure sources such as leaded gasoline and residential $\mathrm{Pb}$-based paint, which are well-known contributors to $\mathrm{Pb}$ contamination of children in many countries. In Japan, as mentioned above, leaded gasoline was completely phased out as early as the mid-1980s, and it is not the custom to paint houses.

$\mathrm{BPb}$ in Japanese children has been reported in a limited number of studies. The first report was by Kaji et al. [9]; the arithmetic mean $\mathrm{BPb}$ of 188 children $(>1$ year) of Shizuoka region in 1993 was $3.16 \mu \mathrm{g} / \mathrm{dL}$ (range $0.80-9.51 \mu \mathrm{g} / \mathrm{dL}$ ). They reported BPb concentration of 23 children aged $<1$ year to be $1.83 \mu \mathrm{g} / \mathrm{dL}$. Kaji [10], in collaboration with Kobayashi and Yoshida [11], reported the arithmetic mean $\mathrm{BPb}$ of 282 children (aged $>1$ year) to be $1.55 \mu \mathrm{g} / \mathrm{dL}$ or GM $1.36 \mu \mathrm{g} / \mathrm{dL}$ (range $0.19-7.68 \mu \mathrm{g} / \mathrm{dL}$ ). They collected blood samples in Shizuoka in 2004-2005. The present GM $(1.15 \mu \mathrm{g} / \mathrm{dL})$ of children in Shizuoka (Table 1) was lower than previously reported values, indicating a general trend for decreasing $\mathrm{Pb}$ exposure in children in Shizuoka.

Based on the $\mathrm{BPb}$ distribution of the present study (Fig. 1), the probability of exceeding the US CDC action level $(10 \mu \mathrm{g} / \mathrm{dL})$ was negligible. It has been claimed that $10 \mu \mathrm{g} / \mathrm{dL}$ is too high a reference $\mathrm{BPb}$, and we postulated a tentative threshold value to be $4 \mu \mathrm{g} / \mathrm{dL}$ based on recent epidemiologic studies, in which significant loss of IQ of children was indicated above $4-5 \mu \mathrm{g} / \mathrm{dL} \mathrm{BPb}[5,6]$. The probability of exceeding this level was $0.075 \%$, indicating that the health risk due to $\mathrm{Pb}$ in Japanese children was minimal, even using the postulated lower threshold value. However, some researchers have claimed that there is no threshold for cognitive effect of $\mathrm{Pb}$ for children [7, 8]. Therefore, it may be desirable to reduce $\mathrm{Pb}$ exposure level of children as much as possible, although the current $\mathrm{Pb}$ exposure level seems to pose minimum risk.

Among the potential factors to vary $\mathrm{BPb}$ of children examined in the present study, age, geographic region, and passive smoking emerged as significant factors, while sex did not.

The negative correlation with age observed in this study is consistent with the results of previous studies. In a number of cohort studies, in which the same children were followed from birth to school age, peak $\mathrm{BPb}$ was observed in the 2nd year of life, followed by decrease thereafter [22-25]. The present result (Table 2) seemed to be consistent with this finding, although ours is a cross-sectional design. This elevation of $\mathrm{BPb}$ in the early years of life has been ascribed to physiological and behavioral features: higher uptake rate of $\mathrm{Pb}$ in digestive tract and more frequent hand-to-mouth activity in early life than in later life. 
Table 3 Comparison of blood $\mathrm{Pb}$ levels of children

\begin{tabular}{lcllll}
\hline & $\begin{array}{l}\text { Number of } \\
\text { samples }\end{array}$ & $\begin{array}{l}\text { Year of } \\
\text { sampling }\end{array}$ & $\begin{array}{l}\text { Age } \\
\text { (years })\end{array}$ & $\begin{array}{l}\text { Geometric mean } \\
\text { BPb } \mu \mathrm{g} / \mathrm{dL}\end{array}$ & Reference \\
\hline Japan & 352 & $2005-2010$ & $1-14$ & 1.07 & This study \\
Canada & 910 & $2008-2009$ & $6-11$ & 0.90 & {$[16]$} \\
USA & 968 & $2005-2006$ & $1-5$ & 1.43 & {$[17]$} \\
& 934 & & $6-11$ & 1.02 & {$[18]$} \\
Germany & 1,560 & $2003-2006$ & $3-14$ & 1.63 & {$[19]$} \\
Korea & 667 & 2008 & $8-11$ & $1.9^{\mathrm{a}}$ & {$[20]$} \\
South Africa & 429 & $2002-2003$ & $5-11$ & $6.4^{\mathrm{a}}$ & {$[21]$} \\
China & 94,778 & $2000-$ & $0-14$ & $8.07^{\mathrm{a}}$ & {$[22]$} \\
India & 754 & 2002 & $<12$ & 8.36 &
\end{tabular}

a Arithmetic mean

This study is the first to examine regional variation in $\mathrm{BPb}$ of children in Japan. As shown in Table 2, GM of BPb in Tokyo was lower than that in Osaka and Shizuoka for all age groups, although statistical significance was seen only for the pair Tokyo-Shizuoka, probably because of small sample size for Osaka, and this result was supported by ANCOVA analysis as well. The lower BPb in Tokyo may partly be related to the smaller percentage of children with passive smoking, whose $\mathrm{BPb}$ was significantly higher than children without it (Fig. 2). The percentage of children with passive smoking was $14.0 \%$ in Tokyo while it was $29.4 \%$ and $20.0 \%$ in Osaka and Shizuoka, respectively, though this difference was not statistically significant $\left(\chi^{2}\right.$ test). However, it may be premature to conclude that there are regional differences in $\mathrm{BPb}$ based on the present study, because sampling was done in only one hospital in Shizuoka and Osaka while blood samples were taken from children in six different clinics/hospitals in Tokyo. Therefore, it is possible that hospital bias was present for Osaka and Shizuoka subjects. The present result should be confirmed by including more samplings and analyses in Shizuoka and Osaka, and also in other regions. Geographic variation, if any, may provide a clue to the source of $\mathrm{Pb}$ for Japanese children.

Higher $\mathrm{BPb}$ in children with passive smoking has been reported in the past $[7,8,26]$ and was ascribed to the fact that cigarette smoke contains $\mathrm{Pb}$ [1]. The present result is consistent with those previous results (Fig. 2). We have estimated the $\mathrm{Pb}$ exposure level of Japanese children living in Tokyo Metropolitan Area by assuming that diet/tap water, soil, house dust, and atmosphere were potential $\mathrm{Pb}$ sources and by summing the exposure level from each medium [27]. The present study suggests that cigarette smoke is another potential source of $\mathrm{Pb}$ for Japanese children which might significantly contribute $(>10 \%$ of absorbed amount) to total $\mathrm{Pb}$ exposure. However, we need to consider the possibility that misclassification of passive smoking status took place in this study: we estimated it based solely on information obtained from questionnaire by asking whether the smoking family member(s) smoked in the presence of the subject child. Future study should include biomarker of cigarette smoke exposure, such as blood or urine cotinine concentration, to relate to $\mathrm{BPb}$ concentration [28].

\section{Conclusions}

Lead concentration in whole blood sampled from Japanese children was analyzed by a contamination-controlled, reliable analytical method. Geometric mean concentration was $1.07 \mu \mathrm{g} / \mathrm{dL}$, indicating that Japanese children are at minimal health risk in terms of the presently recognized safety level. Variation was found in blood lead concentration due to age, region of blood sampling, and passive smoking status. Reduction of passive smoking is an effective practical measure to further reduce lead exposure level, and consequently health risk, of children, since it is postulated that adverse effects of $\mathrm{Pb}$ may not have a threshold.

Acknowledgments The authors appreciate the following collaborating pediatricians:.Drs. A. Uematsu, K. Sugihara, M. Goto, K. Nakamura, H. Koyanagi, K. Baba, H. Utsumi, and H. Yamakage. The authors sincerely appreciate the subject children and their caretakers. This study was supported by a grant from the Food Safety Commission, Cabinet Office, Japan (project \#0801).

\section{References}

1. IPCS. Environmental Health Criteria 165 inorganic lead. Geneva: WHO; 1995.

2. Pocock SJ, Smith M, Baghurst P. Environmental lead and children's intelligence: a systematic review of the epidemiological evidence. BMJ. 1994;309:1189-97.

3. Miranda ML, Kim D, Galeano MAO, Paul CJ, Hull AP, Morgan SP. The relationship between early childhood blood lead levels and performance on end-of-grade tests. Environ Health Perspect. 2007;115:1242-7.

4. Needleman HL, Riess JA, Tobin MJ, Biesecker GE, Greenhouse JB. Bone lead levels and delinquent behavior. JAMA. 1996;275: 363-9. 
5. Surkan PJ, Zhang A, Trachtenberg F, Daniel DB, McKinlay S, Bellinger DC. Neuropsychological function in children with blood lead levels $<10 \mu \mathrm{g} / \mathrm{dL}$. Neurotoxicology. 2007;28:1170-7.

6. Jusko TA, Henderson CR Jr, Lanphear BP, Cory-Slecha DA, Parsons PJ, Canfield RL. Blood lead concentrations $<10 \mu \mathrm{g} / \mathrm{dL}$ and child intelligence at 6 years of age. Environ Health Perspect. 2008;116:243-8.

7. Schwartz J. Low-level lead exposure and children's IQ: a metaanalysis and search for a threshold. Environ Res. 1994;65:42-55.

8. Lanphear BP, Hornung R, Khoury J, Yolton K, Baghurst P, Bellinger DC, et al. Low-level environmental lead exposure and children's intellectual function: an international pooled analysis. Environ Health Perspect. 2005;113:894-9.

9. Kaji M, Gotoh M, Takagi Y, Masuda H. Blood lead levels in Japanese children: effects of passive smoking. Jpn J Pediatr. 1997;101:1584-7. [in Japanese].

10. Kaji M. Blood lead levels in Japanese children-effects of passive smoking. Biomed Res Trace Elem. 2007;18:199-203.

11. Kobayashi N, Yoshida K. Health risk assessment of lead in Japanese children based on blood lead concentration. Jpn J Hyg. 2006;61:295. [in Japanese].

12. $\mathrm{Su}$ M, Barrueto F Jr, Hoffman RS. Childhood lead poisoning from paint chips: a continuing problem. J Urban Health. 2002;79: 491-501.

13. Lanphear BP, Matte TD, Rogers J, Clickner RP, Dietz B, Bornschein RL, et al. The contribution of lead contaminated house dust and residential soil to children's blood lead levels. Environ Res. 1998;79:51-68.

14. Okamoto K, Fuwa K. Low-contamination digestion bomb method using Teflon double vessel for biological materials. Anal Chem. 1984;56:1758-60.

15. Bushnik T, Haines D, Levallois P, Levesque J, Van Oostdam J, Viau C. Lead and bisphenol A concentrations in the Canadian population. Health Rep. 2010;21:7-18.

16. US Center for Disease Control and Prevention. Fourth National Report on human exposure to environmental chemicals. Atlanta, GA: CDC; 2010.

17. Kolossa-Gehring M, Becker K, Conrad A, Lüdecke A, Riedel S, Seiwert M, et al. German Environmental Survey for Children (GerES IV)—first results. Int J Hyg Environ Health. 2007;210: 535-40.
18. Cho S-C, Kim B-N, Hong Y-C, Shin Y-C, Yoo HJ, Kim J-W, et al. Effects of environmental exposure to lead and tobacco smoke on inattentive and hyperactive symptoms and neurocognitive performance in children. J Child Psychol Psychiat. 2010; $51: 1050-7$

19. Mathee A, Röllin H, von Schirnding Y, Levin J, Naik I. Reduction in blood lead levels among school children following the introduction of unleaded petrol in South Africa. Environ Res. 2006;100:319-22.

20. He K, Wang S, Zhang J. Blood lead levels of children and its trend in China. Sci Total Environ. 2009;407:3986-93.

21. Nichani V, Li W-I, Smith MA, Noonan G, Kulkarni M, Kodavor $\mathrm{M}$, et al. Blood lead levels in children after phase-out of leaded gasoline in Bombay, India. Sci Total Environ. 2006;363:95-106.

22. Baghurst PA, McMichael AJ, Wigg NR, Vimpani GV, Robertson EF, Roberts RJ, et al. Environmental exposure to lead and children's intelligence at the age of seven years. N Engl J Med. 1992;327:1279-84.

23. Dietrich KN, Berger OG, Succop PA. Lead exposure and the motor developmental status of urban six-year-old children in the Cincinnati prospective study. Pediatrics. 1993;91:301-7.

24. Canfield RL, Henderson CR Jr, Cory-Slechta DA, Cox C, Jusko TA, Lanphear BP. Intellectual impairment in children with blood lead concentrations below $10 \mu \mathrm{g}$ per deciliter. N Engl J Med. 2003;348:1517-26.

25. Schnaas L, Rothenberg SJ, Flores M-F, Martinez S, Hernández C, Osorio E, et al. Blood lead secular trend in a cohort of children in Mexico City (1987-2002). Environ Health Perspect. 2004;112: $1110-5$.

26. Strömberg U, Lundh T, Schütz A, Skerfving S. Yearly measurements of blood lead in Swedish children since 1978: an update focusing on the petrol lead free period 1995-2001. Occup Environ Med. 2003;60:370-2.

27. Aung NN, Yoshinaga J, Takahashi J. Exposure assessment of lead among Japanese children. Environ Health Prevent Med. 2004; 9:257-61.

28. Mannino DM, Albalak R, Grosse S, Repace J. Second-hand smoke exposure and blood lead levels in U.S. children. Epidemiology. 2003;14:719-27. 